Silvério-Lopes et al., Afr J Tradit Complement Altern Med., (2018) 15 (2): 51-57

https://doi.org/10.21010/ajtcam.v15i2.7

\title{
IMMEDIATE RESPONSE GAIN IN HANDGRIP STRENGTH WITH ACUPUNCTURE: AN EXPERIMENTAL STUDY
}

\author{
Sandra Silvério-Lopes ${ }^{1 *}$, Maria Paula Gonçalves da Mota ${ }^{2}$
}

${ }^{1}$ IBRATE Technology College Rua Voluntários da Pátria, 215 - Curitiba, Paraná, Brasil; ${ }^{2}$ University of Tras-os-Montes and Alto Douro, Research Centre in Sports Science, Health and Human Development (CIDESD) - Quinta de Prados, 5000801, Vila Real - Portugal.

*Corresponding Author Email: san.silverio@yahoo.com.br

\begin{tabular}{|l|}
\hline \multicolumn{1}{|c|}{ Article History } \\
\hline Received: June. 09, 2016 \\
Revised Received: Dec. 12, 2017 \\
Accepted: Dec. 13, 2017 \\
Published Online: Feb. 23, 2018 \\
\hline
\end{tabular}

\begin{abstract}
Background: Muscle strength is an important component for performing different daily tasks and has significant effect on health. Grip strength can be considered as one of the parameters of the overall strength state of the individuals. This study aimed to evaluate immediate response of the capacity to gain handgrip strength after the application of acupuncture.

Materials and Methods: The study was experimental, quantitative, and partially blind clinical trial with a control group. Sample consisted in 73 healthy volunteers of both sexes, not athletes, with average age $35 \pm 10.01$ years, which were divided by deterministic allocation with sequential alternation in three groups: acupuncture (GACP $n=24)$, sham acupuncture (Gsham $\mathrm{n}=25)$ and control (GCRT $\mathrm{n}=24)$. Calibrated mechanical manual dynamometer and needles $0.25 \mathrm{x}$ $40 \mathrm{~mm}$ were used. The handgrip test was performed in all groups, in both hands. Average measures were used for statistical analysis, a procedure done before and after the intervention. GACP received needles in TE5 (Waiguan), ST36 (Zusanli) and GB34 (Yanglingquan). The Gsham received superficial needles placed out of the acupoints.

Results: GACP showed a significant strength gain of $4.78 \mathrm{Kgf}(p=0.005)$, Gsham showed a non-significant gain of $1.13 \mathrm{Kgf}(p=0.370)$ and GCRT that did not receive acupuncture intervention showed a non-significant reduction of handgrip strength average of about $1.97 \mathrm{Kgf}(p=0.210)$.

Conclusion: Acupuncture in a single intervention was able to promote an immediate significant average gain strength response in handgrip of $4.78 \mathrm{Kgf}$.
\end{abstract}

Keywords: Acupuncture, strength gain, handgrip.

\section{Introduction}

The hand is a complex organ with specialized mechanisms that allow men to achieve details and unique activities. Muscular action in hand movements enables a wide range of amplitude and mio-articular arrangements. This makes the hand not only a structure capable of performing great grip strength but, also, presenting fine motor skills such as playing an instrument (Taekema et al., 2009).

The term muscle strength is generally used to define the ability of a particular muscle to produce or to resist a load, and it is classified as isometric, isotonic and isokinetic (Borges et al., 2009). Muscle strength is essential to motor skills performance, whether related to athletic performance or daily life activities (Sasaki et al., 2007).

There is a close relationship between muscle strength and functional capacity. Therefore, the evaluation of muscle strength is of great importance and essential to detect possible changes and risks in specific populations, such as in athletes (Soares et al., 2012).

The precise measurement of the exerted forces by the fingers and hand is of great value to biomechanical studies. It is also useful for evaluating the recovery of people undergoing surgery of the hands or the loss of upper limb mobility due to illnesses, accidents or disabilities (Martins et al, 2015). In their studies, Smith et al. (2006) and Habibi et al. (2013) concluded that, by evaluating the handgrip strength, it is possible to predict the quality of the strength of the whole body, thus becoming an important predictive method.

In the sports area, grip strength is strongly required in specific modalities and can be a key factor to athletic performance, such as athletics throwing, gymnastics, rowing, tennis and others. The occurrence of muscular fatigue in gripping in some decisive moments can result in an error, leading to the athlete to defeat (Leyk et al., 2007). 
Acupuncture is currently being used in clinical practice to improve muscle weakness conditions, lassitude and asthenia, because of it's energetic or invigorating effects, arousing the interest by its study. Based on this fact, some acupoints have awoken research interest for their ergogenic effects (Yin et al., 2007; Ahmedov, 2010). Acupuncture in the clinical setting requires syndromic diagnosis and needs subsequent sessions to achieve results (Maciocia, 2015). A Few studies addressed the immediate response of the ergogenic effects of acute intervention by acupuncture. This study aimed to evaluate immediate response to grip strength gain capacity after a single application of acupuncture.

\section{Materials and Methods Participants and sample}

Research Ethics Committee from IBRATE Technology College approved this study under registration number: 129.429/2012. Sample consisted of 73 sedentary volunteers of both sexes with age average of $35 \pm 10.01$ years old. Volunteers were divided by deterministic allocation in sequential alternation into three groups: acupuncture $($ GACP $n=24)$, sham acupuncture $($ Gsham $n=25)$ and control (GCRT $n=24)$.

Volunteers were recruited by self-selection (posters) meeting the following eligibility criteria: not being pregnant, no surgeries in upper limbs, no chronic musculoskeletal disorders that could interfere with the correct execution of the dynamometer test. Also, volunteers could not have ingested analgesics, antispasmodic, antiinflammatory, muscle relaxant or anesthetic medications, received acupuncture or physiotherapy within three days before the data collection. Volunteers should not have done physical exercise to strengthen upper limbs during the week before the data collection.

\section{Methods}

The study was blinded by the evaluator and partially blinded by volunteers, quantitative and with a control group. Data collection, acupuncture and handgrip test were conducted on the premises of the clinical school of IBRATE Technology College (Curitiba, Brazil).

After signing a consent term, according to the Helsinki Declaration, volunteers were designated by deterministic allocation in sequential alternation to one of three groups: acupuncture (GACP $\mathrm{n}=24)$, sham group (Gsham $\mathrm{n}=25)$ and control group (GCRT $\mathrm{n}=24)$, with no exclusion in the sample until the end of the study.

In the first stage of the study, volunteers performed the grip strength test, using a mechanical manual dynamometer (Grip dynamometer, model 5001, Takei Scientific Instruments, Japan), previously calibrated. Equipment measures strength depending on the amount of tension produced, ranging from 0 to $100 \mathrm{Kgf}$, with individual adjustment size of the handle strap (Figure 1).

For a correct application of the handgrip strength test, we followed the protocol recommended by Fernandes and Marins (2011). It consists in communication of guidelines to the volunteer about the correct position for performing the test, number of measurements, rest period between attempts, duration of contraction time, and strap position.

Volunteers were informed that they should wait for the evaluator's command to start performing each maximum gripping movement. They performed three repetitions of maximum strength contractions using the right hand and another three using the left hand. For the statistical evaluation, the average of the three measurements of each hand, before and after the intervention, were considered.

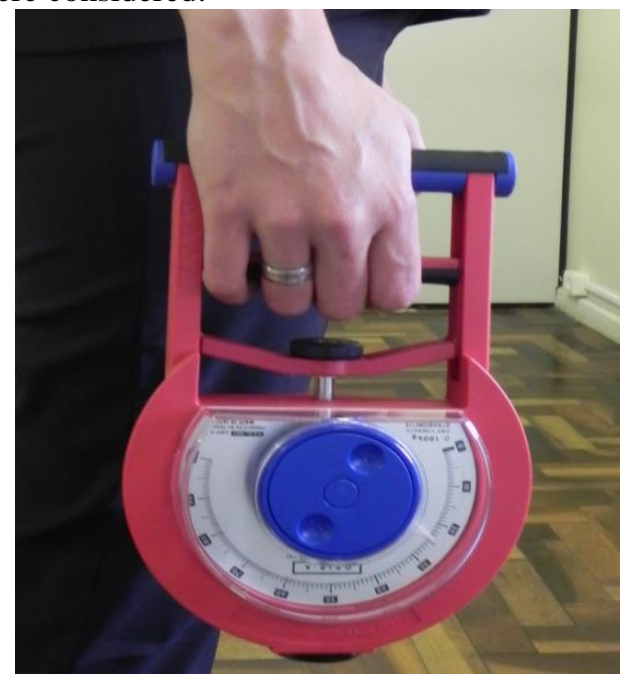

Figure 1: Illustration of the handgrip test 
The maximum contraction time was three seconds, after when the evaluator voiced the "stop" command. With the dynamometer pointer locked at the maximum value, the strength value was read from the device.

The dynamometry test was applied by the invitation of a professional Physical Education, who was not aware to which group each volunteer belonged. After running the test, volunteers belonging to the GACP were needled bilaterally in the following acupoints; ST36 (Zusanli), TE5 (Waiguan) and GB34 (Yanglingquan), according to Lian et al. (2012), and they remained at rest for 20 minutes. Disposable stainless steel threadlike needles, measuring 0.25 x 40 mm (Arhon Din ${ }^{\circledR}$, Brazil) were used.

Among the above-mentioned acupoints, ST36 is considered the most effective to gain strength by acupuncture (Ahmedov, 2010). Also, Maciocia (2015) recommended acupoint TE5 to increase "Yang energy" and vitality, and GB34 to strengthen the tendons.

Disposable needles were introduced at a depth of 1.5 inches $(38.10 \mathrm{~mm})$ and initially stimulated until the patient reported a "deqi" feeling, which corresponds to a tingling, light "shock" and/or heavy feeling in the arms. The Gsham group received only superficial needling and outside the acupoints. A distant location was chosen medially 20 $\mathrm{mm}$ far from those points used in the GACP. GCRT was submitted to the handgrip strength test, and remained at rest for 20 minutes.

All the acupuncture applications were performed by a professional trained acupuncture. Volunteers did not know to which group they belonged. After the resting period, all the volunteers of all groups were reevaluated with the grip strength test.

\section{Statistical analysis}

Data collected were subjected to an exploratory analysis, using graphical methods of Box-and-Whiskers and Steam and Leaf for the identification and deletion of outliers that potentially influenced the central tendency parameters (Elliott and Marsh, 2008). Symmetry and flattening of the distribution curves were evaluated by means of the skewness and kurtosis values, respectively. Distribution normality was confirmed by the Kolmogorov-Smirnov non-parametric test, with the Lilliefors correction.

Next, using descriptive statistics, average and standard deviation of the variables under study were calculated, for the total sample and for each study group. Comparison between mean values obtained for each group at each moment of the evaluation was made by One-Way ANOVA test with Post Hoc LSD to compare variables in pairs. Intragroup analysis compared the average of the force between the pre-treatment times and immediate post-treatment using the Student t-test for paired samples, and significance level was set to 0.05 . For all the statistical analysis, we used SPSS program, version 20

\section{Results}

Table 1 shows average values $( \pm \mathrm{SD})$ by age and handgrip strength for each group at each time of evaluation.

Table 1: Average values $( \pm \mathrm{SD})$ for age and handgrip strength (Kgf) in the GACP, Gsham and GCRT at every moment of the evaluation

\begin{tabular}{ccccc}
\hline \multirow{2}{*}{ Group } & \multirow{2}{*}{ n } & \multirow{2}{*}{ Average age } & \multicolumn{2}{c}{ Handgrip strength } \\
\cline { 4 - 5 } & & & Pre-treatment & Immediate post \\
\hline GACP & 24 & $36.42 \pm 10.74$ & $32.21 \pm 9.71$ & $33.64 \pm 9.93$ \\
Gsham & 25 & $33.48 \pm 7.57$ & $34.65 \pm 8.71$ & $34.99 \pm 8.76$ \\
GCRT & 24 & $30.92 \pm 9.83$ & $32.95 \pm 10.40$ & $32.44 \pm 10.75$ \\
\hline
\end{tabular}

No significant differences in age between groups were found. Variation percentage between groups revealed significant differences in handgrip strength of the pre-treatment time to the immediate post-treatment time $(\mathrm{F}=6.183 \mathrm{p}$ $=0.003$ ), see Figure 2 .

When comparing groups in pairs, regarding the percentage variations, the GACP showed significant increase in strength when compared to GCRT $(\mathrm{p}=0.001)$ and, also, a statistically significant trend when compared to Gsham ( $\mathrm{p}$ $=0.059$ ). There was no significant difference between Gsham and GCRT.

When comparing strength average between pre-treatment and immediately post-treatment moments in each group, only the GACP presented significant increase in the handgrip strength $(\mathrm{p}=0.005)$. 


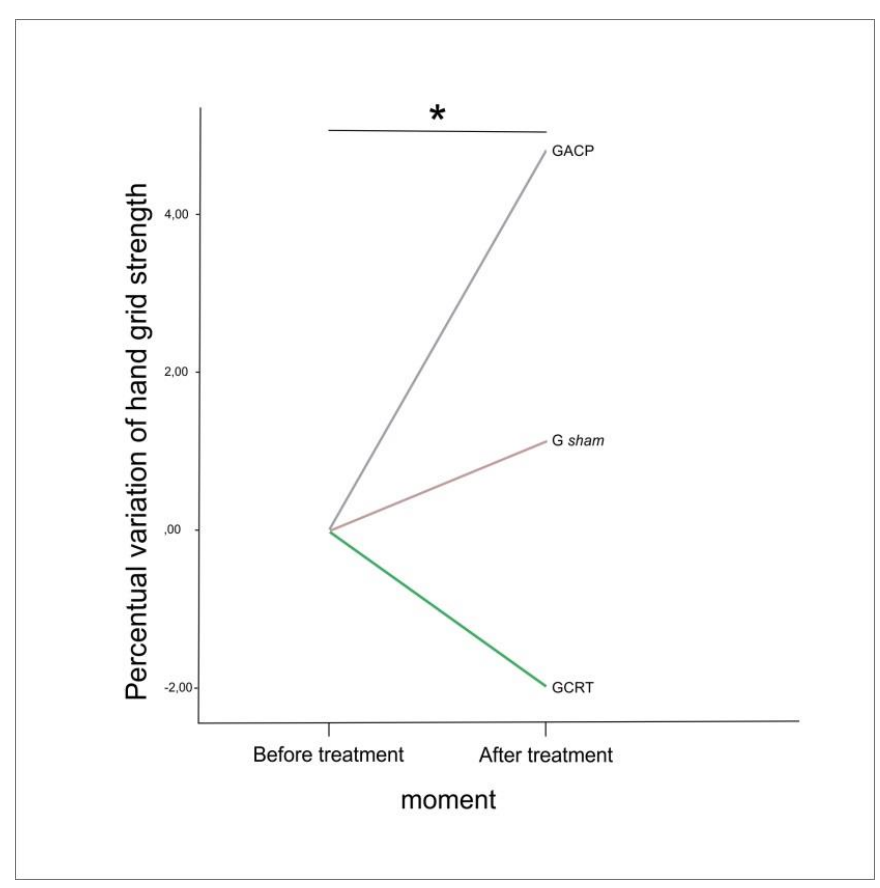

Figure 2: Percentage variation of the average handgrip strength (Kgf) in the GACP (ACP), Gsham (SHAM) and GCRT (CONT), between the two moments of evaluation $(* \mathrm{p}<0.05)$.

\section{Discussion}

This study raised an important result regarding to the immediate effect of acupuncture in a single intervention. Results indicate that acupuncture was able to significantly and immediately increase gain of handgrip strength measured by the dynamometer. This result is consistent with the studies of Yim et al (2007) and Huang et al. (2007) who concluded that there are ergogenic effects of some acupoints. It is possible that the lost of strength found in GCRT may be due to muscle relaxation, considering that the volunteers of this group received no intervention. However, they rested supine on a stretcher at the same time and under the same conditions and between other subjects.

Muscular strength is among the key components for the evaluation of fitness. According Lucareli et al (2010) handgrip test can be considered as a parameter of the overall power status of individuals having high acceptability in physical fitness tests, particularly when evaluating muscle strength of the upper members. Therefore, to assess handgrip strength it's important and very frequent in occupational health, driver's licenses of expedition expertise, according to the National Traffic Council (2012), and admission tests in various types of work, among others.

In the present study we observed the appropriate use of the dynamometer as an assessment instrument between pre and post-treatment moments. However, it is important to notice that a methodical and meticulous execution is required by the evaluator, with verbal commands and demonstration. It is not unusual for volunteers to try to go beyond their own limits as to compete with themselves.

Handgrip strength is influenced by factors such as age, gender, weight, palm surface and fingers size, and body mass index, among others (Habibi et al., 2013). In the present study, groups were homogeneous.

There are 35 muscles related to forearm and hand movement, and many of them are involved in the grip action. According to Kisner and Colby (2009) the muscles of the flexor mechanism of the hand and forearm create grip strength, while the forearm extensors stabilize the wrist. Steven-Moore (2002), studying pathogenesis models of repetitive stress injuries in extremities of upper limbs, confirms that the main critical factor for the development of this type of injury is the compression force transmitted to the retinaculum extensor muscle. Therefore, handgrip strength gains are important, supporting the curative, preventive or performance aspects.

Nichols and Harrigan (2006) point out that the use of complementary and alternative medicine (CAM) among western athletes is increasing. Alternative therapies, including acupuncture, are used by $56 \%$ of athletes, against $36 \%$ in the non-athlete population. Koh et al. (2012) demonstrate concern about their studies because the athletes are among the largest users of CAM, including looking for "on their own" resources and, opposed to the rest of the population, they would be using these therapies not only for rehabilitation, but also aiming potential benefits in sports performance, often without proper monitoring.

Recent years have witnessed a growing interest of the scientific community in the possible effects of acupuncture in the variables involved in the physical performance (Zhou et al., 2012). Studies of physical strength and performance are more evident in athletes. A study by Luna and Fernandes-Filho (2005) evaluated the effects of 
acupuncture in high-performance speedsters in maximal dynamic strength variables, explosive, anaerobic endurance and speed. The results showed a clinically significant improvement in all the variables studied and statistically significant improvement in maximal dynamic strength and explosive power. In that study they do not considered the sham and control groups.

Hubscher et al. (2010) evaluated the effect of acupuncture in the isometric strength of hip in 33 nonprofessional athletes. They found significant strength gains compared to the sham and the placebo group. Another study of Snyder et al. (2011) in healthy subjects demonstrated that acupuncture at the ST36 point (Zusanli) increased significantly propulsion force of vertical jumps. In another area, Rancan et al. (2009) also found good results in strength gains, concluding that the acupuncture has increased molar bite strength and changes in electromyography activity of masticatory muscles in the volunteers assessed.

Overall, the present study and the literature cited converge to a consensus, strongly indicating the good results, the use of acupuncture to gain strength, despite targeting different muscle groups that proposed methodology, strength type and population. However, it is worth to notice that there are results that contradict those discovered in this study, such as Costa and Araújo (2008), conducted in mice, not in humans. They found that the isometric strength of the tibialis anterior muscle was reduced after acupuncture.

Ergogenic effects physiology associated with acupuncture has been the focus of some authors. Lee et al. (2002) through a study with rats showed suppressive effects of acupuncture on 5 HT (5-hydroxytryptamine) and the expression of TPH (tryptophan hydroxylase) in the dorsal raphe nucleus, observing that the increased concentration of 5 HT reduces physical performance in humans and rats.

A study of Toda (2012) concluded that acupuncture was able to increase carnitine levels and, therefore, decrease the fatigue of skeletal muscle. It is known that a muscle fatigue tend to lose strength, particularly to sustain the movement. Enoka and Duchateau (2007) also found functional improvement using acupuncture, by increasing the local microcirculation and, consequently, better oxygenation of the muscle tissues, as well as metabolites removal and increase of ATP synthesis (Sandner-Kiesling et al., 2001; Goldman et al., 2010). Nowadays there are also authors who study ways to improve acupuncture techniques to enhance their therapeutic effects (Su-ll In et al., 2016).

Increase in muscle strength or other physical capabilities are important, making them crucial to certain populations, even small gains. Recalling the London Olympics Games in 2012, gold, silver and bronze medals in the men's weightlifting up to $105 \mathrm{~kg}$ were separated by a difference performance of only $1 \mathrm{Kg}$ (http://www.olympic.org/weightlifting, Acess: 05/22/2015).

Another area that can be covered with the strength gain benefits is gerontology. Alexander et al. (2008) studied the behavior of grip muscle strength in elderly. Observing that this population loses muscle mass and consequent muscle strength over the years, they concluded that muscle strength is a physical capability of extreme importance in domestic accidents.

About the composition of acupuncture points assessed in this study, we observed that, in addition to the ergogenic effects, there was operational ease of execution, good tolerance and openness of the volunteers, which provide therefore a recommendation for further work and physical training programs.

\section{Conclusion}

Results of this study demonstrated significant and immediate increase of grip strength when comparing the moments before and after the application of acupuncture needles in the points: ST36 (Zusanli), TE5 (Waiguan), and GB34 (Yanglingquan). Research findings suggest exciting possibilities, through discreet. It is recommended to extend this study to evaluate efficacy and operational viabilities of use in athlete monitoring programs, particularly for highperformance athletes, in which small gains may be representative for the final result. Gain Stability evaluation about over time gain is suggested for future studies.

Conflict of Interest: The authors declare that they have no conflict of interests.

\section{References}

1. Ahmedov, O.S. (2010). Ergogenic effect of acupuncture in sport and exercise: a brief review. The Journal of Strength \& Conditioning Research. 24(5):1421-1427.

2. Borges, Jr N.G., Domenech, S.C., Silva, A.C.K., Dias, J.A. and Sagawa, Jr Y. (2009). Comparative study of maximum isometric grip strength in different sports. Revista Brasileira de Cineantropometria e Desempenho Humano. 11(3): 292-298.

3. Costa, L.A. and Araújo, J.E. (2008). The immediate effects of local and adjacent acupuncture on the tibialis anterior muscle: a human study. Chinese Medicine. 3(17):1-6.

4. Elliott, J. and Marsh, C. (2008). Exploring Data: An introduction to Data Analysis for Social Scientists. 2nd edition. Polity Press. 
5. Enoka, R.M. and Duchateau, J. (2007). Muscle fatigue: what, why and how it influences muscle function. The Journal of Physiology. 586(1):11-23.

6. Fernandes, A.A. and Marins, J.C.B. (2011). Test of handgrip strength: a methodological analysis and normative data in athletes. Fisioterapia em Movimento. 24(3): 567-578.

7. Goldman, N., Chen, M., Fujita, T., Xu, Q., Peng, W., Liu, W., Jensen, T.K., Pei, Y., Wang, F., Han, X., Chen, J.F., Schnermann, J., Takano, T., Bekar, L., Tieu, K. and Nedergaard, M. (2010). Adenosine A1 receptors mediate local anti-nociceptive effects of acupuncture. Nature Neuroscience. 13(7):883-888.

8. Habibi, E., Kazemi, M., Dehghan, H., Mahaki, B. and Hassanzadeh, A. (2013). Handgrip and pinch strength: effects of workload, hand dominance, age, and body mass index. Pakistan Journal of Medical Science. 29(1suppl.): 363-367.

9. Huang, L., Zhou, S., Lu, Z., Tian, Q. and Li, X. (2007). Bilateral effect of unilateral electroacupuncture on muscle strength. J Altern Complement Med. 13(5):539-546.

10. Hübscher, M., Vogt, L., Ziebart, T. and Banzer, W. (2010). Immediate effects of acupuncture on strength performance: a randomized, controlled crossover trial. Europian Journal of Applied Physiology. 110(2):353-358.

11. Su-1l In, Gwak Y.S., Kim H.R., Razzag A., Lee K-S., Kim H.Y., Chang S.C., Lee B.H., Grimes C.A., Yang C.H. Hierarchical Micro/Nano-Porous Acupuncture Needles Offering Enhanced Therapeutic Properties. Science Reports, 6, 34061; doi: 10.1038/srep34061 (2016).

12. International Olympic Committee. Results of London Olympics - Weightlifting. Available in: http://www.olympic.org/weightlifting. Acess: 05/22/2015.

13. Kisner, C. and Colby, L.A. (2009). Therapeutic Exercise: Foundations and Techniques. $5^{\text {th }}$ edition. Philadelphia, USA: F.A. Davis Company.

14. Koh, B., Freeman, L. and Zaslawski, C. (2012). Alternative medicine and doping in sports. Australasian Medical Journal. 5(1):18-25.

15. Lee, S.H., Chung, S.H., Lee, J.S., Kim, S.S., Shin, H.D., Lim, B.V., Jang, M.H., Kim, H., Kim, E.H. and Kim, C.J. (2002). Effects of acupuncture on the 5-hydroxytryptamine synthesis and tryptophan hydroxylase expression in the dorsal raphe of exercised rats. Neuroscience Letters. 332(1):17-20.

16. Leyk, D., Gorges, W., Ridder, D., Wunderlich, M., Ruther, T., Sievert, A. and Essfeld, D. (2007). Hand-grip strength of young men, women and highly trained female athletes. European Journal of Applied Physiology. 99(4):415-21.

17. Lian, Y.L., Chen, C.Y., Hammes, M. and Kol, B.C. (2012). Pictorial atlas of acupuncture - An illustrated manual of acupuncture points. Potsdam, Germany: H.F. Ullmann.

18. Lucareli, P.R.G., Lima, M.O., Lima, F.P.S., Gimenes, R.O., Lucareli, J.G.A., Garbelotti, Jr S.A., Fukuda, T.Y. and Pompeu, J.E. (2010). Comparison of methods of measurement of the finger flexor muscles strength through dynamometry and modified manual sphygmomanometer. Einstein. 8(2): 205-208.

19. Luna, M.P. and Fernandes-Filho, J. (2005). Effects of the acupuncture in the performance of high performance sprinter athletes of Rio de Janeiro. Fitness \& Performance Journal. 4:199-214.

20. Maciocia G. (2015). The Foundations of Chinese Medicine: A Comprehensive text for acupuncturists and Herbalists. 2nd edition. Churchill Livingstone, Elsevier.

21. Martins, J.C., Teixeira-Salmela, L.F., Aguiar, L. T., Souza, L.A.C., Lara, E.M. and Faria, C.D.C.M. (2015). Assessment of the strength of the trunk and upper limb muscles in stroke subjects with portable dynamometry: a literature review. Fisioterapia em Movimento. 28(1):169-186.

22. National Traffic Council. Resolution CONTRAN n. 425. (2012) Available in: http://www.denatran.gov.br. It provides for the examination of physical and mental ability, psychological evaluation and accreditation of public and private organizations that treat the art. 147, I, $\S \S 1$ to 4 and art. 148 of the Brazilian Traffic Code. Acess: 06/05/2012.

23. Nichols, A.W. and Harrigan, R. (2006). Complementary and alternative medicine usage by inter-collegiate athletes. Clinical Journal of Sport Medicine. 16(3):232-237.

24. Rancan, S.V., Bataglion, C., Bataglion, S.A., Bechara, O.M., Semprini, M., Siéssere, S., Sousa, J.P., Crippa, J.A., Hallak, J.E. and Regalo, S.C. (2009). Acupuncture and Temporomandibular Disorders: A 3-Month Follow-up EMG Study. Journal of Alternative and Complementary Medicine. 15(2):1307-1310.

25. Sandner-Kiesling, A., Litscher, G., Voit-Augustin, H., James, R.L. and Schwarz, G. (2001). Laser doppler flowmetry in combined needle acupuncture and moxibustion: a pilot study in healthy adults. Lasers in Medical Science. 16(3):184-191.

26. Sasaki, H., Kasagi, F., Yamada, M. and Fujita, S. (2007). Grip strength predicts cause-specific mortality in middleaged and elderly persons. American Journal of Medicine. 120(4):337-42.

27. Smith, T.T., Smith, S.W., Martin, M., Henry, R., Weeks, S. and Bryant, A. (2006). Grip strength in relation to overall strength and functional capacity in very old and oldest old females. Physical and Occupational Therapy in Geriatrics. 24(4): 63-78.

28. Snyder, B.J., McGary, B.D.C., Molnar, S. and Wyant, A. (2011). The effect of TCM acupuncture on vertical jump 
height enhancement. Journal of Chiropractics Education. 25(1):9.

29. Soares, A.V., Carvalho Jr, J.M., Fachini, J., Domenech, S.C. and Borges Jr, N.G. (2012). Correlation between handgrip, scapular and lumbar dynamometry tests. Revista Acta Brasileira do Movimento Humano. 2(1): 5-72.

30. Steven-Moore J.S. (2002). Biomechanical models for the pathogenesis of specific distal upper extremity disorders. American Journal of Industrial Medicine. 41(5):353-369.

31. Taekema, D.G., Gussekloo, J., Maier, A.B., Westendorp, R.G.J. and Craen, A.J.M. (2010). Handgrip strength as a predictor of functional, psychological and social health. A prospective population-based study among the oldest old. Age and Ageing. 39(3):331-337.

32. Toda S. (2012). Effects of acupuncture on carnitine for skeletal muscle fatigue. Chinese Medicine. 3(1):9-12.

33. Yim, Y.K., Lee, H., Hong, K.E., Kim, Y.I.I., Lee, B.R., Son, C.G. and Kim, J.E. (2007). Electro-acupuncture at acupoint ST36 reduces inflammation and regulates immune activity in collagen-induced arthritic mice. EvidenceBased Complementary and Alternative Medicine. 4(1): 51-57.

34. Zhou, S., Huang, L.P., Liu, J., Yu, J.H., Tian, Q. and Cao, L.J. (2012). Bilateral effects of 6 weeks unilateral acupuncture and electroacupuncture on ankle dorsiflexors muscle strength: a pilot study. Archives of Physical Medicine and Rehabilitation. 93(1):50. 